\title{
AVALIAÇÃO DE USUÁRIOS DE CRACK SOBRE OS GRUPOS DE FAMILIARES NO CENTRO DE ATENÇÃO PSICOSSOCIAL*
}

Maria de Lourdes Custódio Duarte', Kelly Ribeiro Viana², Agnes Olschowsky

${ }^{1}$ Enfermeira. Doutora em Enfermagem. Professora da Universidade Federal do Rio Grande do Sul. Porto Alegre-RS-Brasil. ${ }^{2}$ Enfermeira. Técnica Administrativa na Escola de Enfermagem da UFRGS. Porto Alegre-RS-Brasil.

RESUMO: A pesquisa buscou avaliar qualitativamente a percepção de usuários de crack sobre grupos de familiares. Trata-se de um recorte de um estudo de avaliação qualitativa realizado no ano de 2012, em um Centro de Atenção Psicossocial Álcool e Drogas do Sul do Brasil. Utilizou-se o referencial teórico da Avaliação de Quarta Geração, tendo como dispositivo metodológico o Círculo Hermenêutico-Dialético, através de observações e entrevistas com doze usuários de crack. Na análise foi utilizado o Método Comparativo Constante, que gerou a unidade de significado Grupo de Família. O grupo foi avaliado pelos usuários como espaço importante para os familiares, servindo como fonte de escuta, desabafo e de informação, instrumentalizando-os na forma de lidar com os usuários no domićlio. Sugere-se que os serviços substitutivos proporcionem horários diversificados de grupos de familiares para fidelizar as famílias nestes espaços, promover a continuidade do tratamento e os efeitos benéficos para o usuário de crack.

DESCRITORES: Grupos de autoajuda; Avaliação de serviços de saúde; Cocaína/crack; Família.

\section{CRACK USERS' EVALUATION REGARDING FAMILY GROUPS IN THE PSYCHOSOCIAL CARE CENTER}

\begin{abstract}
This study sought to qualitatively evaluate the perception of crack users regarding family groups. It is an excerpt from a qualitative study undertaken in 2012 in a Psychosocial Care Center - Alcohol and Drugs, in the South of Brazil. It used the theoretical framework of Fourth Generation Evaluation, with the Hermeneutic-Dialectic Circle as its methodological device, through observations and interviews held with 12 crack users. The Constant Comparative Method was used in the analysis, which generated the unit of meaning of Family Group. The group was evaluated by the crack users as an important space for the family members, serving as a source of listening, relieving feelings by expressing grievances, information, and instrumentalizing them in how to deal with the crack users in the home. It is suggested that the substitute services should make varying hours available for family groups, so as to loyalize the families in these spaces and promote the continuity of the treatment and the beneficial effects for the crack users.
\end{abstract}

DESCRIPTORS: Self-help groups; Evaluation of health services; Crack cocaine; Family.

\section{EVALUACIÓN DE USUARIOS DE CRACK SOBRE LOS GRUPOS DE FAMILIARES EN EL CENTRO DE ATENCIÓN PSICOSOCIAL}

RESUMEN: La investigación tuvo la finalidad de evaluar cualitativamente la percepción de usuarios de crack sobre grupos de familiares. Trae una parte de un estudio de evaluación cualitativa realizado en el año de 2012, en un Centro de Atención Psicosocial de Álcohol y Drogas del Sur de Brasil. Fue utilizado el referencial teórico da Evaluación de Cuarta Generación, teniendo como dispositivo metodológico el Círculo Hermenéutico-Dialético, a través de observaciones y entrevistas con doce usuarios de crack. En el análisis, fue utilizado el Método Comparativo Constante, que generó la unidad de significado Grupo de Familia. El grupo fue evaluado por los usuarios como espacio importante para los familiares, siendo una fuente de escucha, desahogo y de información, instrumentalizándolos para relacionarse con los usuarios en domicilio. Se sugiere que los servicios sustitutivos proporcionen horarios diversificados de grupos de familiares para fidelizar las familias en estes espacios, promover la continuidad del tratamiento y los efectos benéficos para el usuario de crack. DESCRIPTORES: Grupos de autoayuda; Evaluación de servicios de salud; Cocaína crack; Familia.

*Artigo extraído da Tese de doutorado intitulada: Familiares dos usuários de crack em um CAPS AD III: avaliação das necessidades de cuidados. Universidade Federal do Rio Grande do Sul, 2013.

Autor Correspondente:

Maria de Lourdes Custódio Duarte

Universidade Federal do Rio Grande do Sul

Rua Gonçalves Ledo, 20 - 90610-250 - Porto Alegre-RS-Brasil

E-mail: malulcd@yahoo.com.br
Recebido: $24 / 08 / 2014$

Finalizado: 04/12/2014 


\section{INTRODUÇÃO}

Atualmente, o crack vem sendo reconhecido como um problema emergente na saúde pública. O mesmo destaca-se pelo seu potente efeito, de curta duração, estimulando a administração repetida, de tal forma que, depois de sua experimentação, deflagra-se o uso intenso e compulsivo, e ele passa a desempenhar papel central na vida do usuário ${ }^{(1-2)}$.

Estimou-se que no Brasil em 2005 havia 380 mil usuários e dependentes de crack, chegando em 2009 a 600 mil. Essa droga apareceu no país de forma progressiva, de fácil acesso e preço baixo, causando dependência e danos físicos rapidamente $^{(3)}$. Em dados relacionados ao uso de drogas ilícitas nos EUA, a cocaína aparece como a principal responsável pelas internações hospitalares, e, dentre as formas de uso da cocaína nesse mesmo país, o crack é a forma mais utilizada ${ }^{(1)}$.

O uso compulsivo do crack interfere e compromete também as questões relativas ao relacionamento social do indivíduo, de modo que os vínculos sociais e familiares se fragilizam e rompem-se, marginalizando-o progressivamente $^{(1)}$. Assim, a dificuldade no convívio entre usuário e familiares é caracterizada como uma das principais dificuldades a serem enfrentadas $^{(4)}$.

Compreende-se que a família possui papel essencial no processo do tratamento, pois quando passa a inserir-se no cotidiano do serviço, consegue compreender o contexto das drogas e suas especificidades, consequentemente entende as necessidades do usuário ${ }^{(5)}$. Possibilita, também, que alguns pré-conceitos sejam quebrados, amplia a visão frente às dificuldades vivenciadas pelas pessoas que usam da droga, buscando uma nova maneira para se lidar com esse usuário de crack.

Ao envolver a família no tratamento do usuário de drogas, dando-lhe suporte para enfrentar as dificuldades inerentes do cotidiano, a carga emocional da família e do próprio usuário é amenizada, aumentando o nível de interação e empatia entre eles ${ }^{(6)}$. É nesse contexto que se inserem os Centros de Atenção Psicossocial Álcool e Drogas (CAPS AD).

O CAPS AD é um ponto de atenção especializada da Rede de Atenção Psicossocial destinado a proporcionar a atenção especializada, integral e contínua às pessoas com necessidades relacionadas ao consumo de álcool, crack e outras drogas. Este tipo de serviço possui, como característica de funcionamento, produzir, em conjunto com o usuário e seus familiares um projeto de tratamento que acompanhe o usuário nos contextos cotidianos, promovendo e ampliando as possibilidades de vida e mediando suas relações sociais ${ }^{(3)}$.

Os CAPS AD é uma estratégia para o acolhimento, prevenção e acompanhamento de pessoas com dependência química ${ }^{(7)}$. Dessa forma, busca-se descentralizar a assistência, promover a articulação social e intersetorial, fortalecendo assim os laços entre o campo da saúde mental e a comunidade.

Dentre as inúmeras estratégias de apoio, de inserção e de estímulo à participação no tratamento do usuário de crack nos CAPS AD, destacam-se os grupos de familiares. A atividade grupal se torna um instrumento essencial para os familiares de usuários de crack, pois os auxilia a conviverem com os problemas em decorrência do uso dessa substância, aprendendo a manejálos de modo mais saudável ${ }^{(7)}$.

Este estudo advém das vivências das pesquisadoras em atividades de extensão, pesquisa e ensino em um CAPS AD, em que a convivência com familiares de usuários de crack no serviço possibilitou trocas sobre a realidade familiar e a importância da participação desse núcleo de cuidado no cotidiano do CAPS e no tratamento do usuário. Assim, tem-se a seguinte questão norteadora: Qual a avaliação de usuários de crack sobre os grupos de familiares que ocorrem no CAPS AD de um município do Sul do país?

Entende-se neste estudo que os grupos de apoio aos familiares ofertados pelos CAPS AD são espaços destinados para o compartilhamento de experiências e sentimentos, possibilitando uma nova percepção da situação, ajudando-os no enfrentamento das dificuldades ocasionadas pelas consequências do uso do crack pelo usuário no seu cotidiano.

Neste estudo apresentar-se-ão dados parciais da pesquisa intitulada "Familiares dos usuários de crack em um CAPS AD III: avaliação das necessidades de cuidados"(8). Neste artigo temse o objetivo de avaliar a percepção de usuários de crack sobre os grupos de apoio de familiares ofertados por um CAPS AD III do Sul do Brasil.

Pretende-se que os resultados deste estudo contribuam no avanço do conhecimento científico no campo da saúde mental, em especial às demandas relacionadas ao consumo de crack 
na região investigada. Espera-se também que a pesquisa possa dar subsídios para avaliar e rediscutir os avanços e desafios dos grupos de familiares, além de problematizar a cobertura e oferta dos cuidados disponibilizados em rede, em consonância com os princípios da Reforma Psiquiátrica e das políticas de saúde mental vigentes no Brasil.

\section{MÉTODO}

Este estudo foi realizado em um CAPS AD tipo III, localizado na fronteira oeste do estado do Rio Grande do Sul.

Trata-se de uma avaliação construtivista, responsiva, com abordagem hermenêuticadialética, denominada Avaliação de Quarta Geração $^{(9)}$. Caracteriza-se, também, por ser descritiva e analítica, com abordagem qualitativa dos dados. É descritiva porque observou, registrou e correlacionou fatos sem manipulá-los.

A Avaliação de Quarta Geração é uma forma de avaliação em que as reivindicações, preocupações e questões do grupo de interesse servem de base para determinar a informação necessária, a qual é implementada através dos pressupostos metodológicos do paradigma construtivista $^{(9)}$. O Círculo Hermenêutico Dialético foi utilizado como técnica de coleta de dados a qual prevê em uma primeira etapa a observação e, posteriormente, as entrevistas. Assim, a coleta de dados ocorreu de $1^{\circ}$ de julho a $1^{\circ}$ de outubro de 2012, sendo realizadas 500 horas de observação no CAPS AD, as quais foram registradas em um diário de campo, e guiadas por um roteiro sobre atendimento aos familiares de usuários de crack, enfocando os grupos de famílias.

Nesse sentido, houve a necessidade, nesta pesquisa, de contar com a participação de quatro observadores externos ao serviço que tinham conhecimentos sobre observação participante ${ }^{(9)}$ , que revezaram-se em todos os turnos a fim de captar maior observação acerca do objeto do estudo.

O serviço estudado, no momento da coleta de dados, ofertava cinco grupos de familiares havia um ano, uma vez por semana, dois pela manhã, dois à noite e um à tarde. Em cada grupo havia em média sete familiares e eram coordenados por uma psicóloga e por uma terapeuta ocupacional.
Por meio da utilização do círculo hermenêutico-dialético, as primeiras entrevistas foram menos estruturadas, permitindo que o respondente falasse livremente sobre os grupos de familiares de usuários de crack. À medida que as entrevistas foram sendo realizadas as análises permitiram a identificação questões expressas nas entrevistas seguintes, de modo que, cada vez mais, as entrevistas se estruturassem sem deixar de permitir que todos os entrevistados pudessem lançar novas questões, se assim o desejassem.

Dos 17 usuários de crack que estavam em tratamento no serviço estudado, foram entrevistados 12, pois apenas esse quantitativo possuía familiares inseridos no CAPS AD no período de coleta de dados e estava de acordo com os critérios de inclusão. Esses usuários autorizaram a gravação das entrevistas. Os critérios de seleção dos participantes: usuários que possuíam seus familiares nos grupos, boas condições de comunicação e maiores de 18 anos.

Os sujeitos que compuseram o estudo foram numerados de 1 a 12, na ordem das entrevistas, e denominados de $U$ (usuário).

As entrevistas foram agendadas, previamente, de acordo com o dia, hora e local marcados pelos próprios entrevistados. Todas foram gravadas e analisadas, a fim de introduzir no círculo hermenêutico questões/assuntos na próxima entrevista. Dessa forma, a primeira entrevista durou 10 minutos e a última, 58 minutos, uma vez que o primeiro entrevistado respondeu apenas uma pergunta disparadora: Qual é a sua avaliação sobre o Grupo de familiares de que seu parente participa? Esse questionamento, ao final do círculo hermenêutico dialético, gerou mais oito perguntas que os próximos participantes foram sendo convidados a responder, ou seja, cada participante foi entrevistado apenas uma vez.

Para a análise dos dados foi utilizado o Método Comparativo Constante, que permite que a análise seja realizada, concomitantemente, à coleta de dados $^{(9)}$, o que gerou os resultados (unidades de significados).

Assim, essas unidades possibilitaram construir a categoria temática "Cuidado", que posteriormente direcionou para a categoria analítica "Cuidado ao familiar de usuário de crack". Nesta categoria analítica discutimos a unidade de significado: "Grupo de família". 
Utilizou-se o Termo de Consentimento Livre e Informado em duas vias, de acordo com a Resolução 196/1996 do Conselho Nacional de Saúde, que institui diretrizes e normas regulamentadoras de pesquisa envolvendo seres humanos ${ }^{(10)}$. Este estudo foi aprovado pelo Comitê de Ética em Pesquisa da Universidade Federal do Rio Grande do Sul (UFRGS), com o Parecer número 75635.

\section{RESULTADOS}

\section{Grupo de Família}

A maioria dos usuários, cerca de $75 \%$, possuía idade entre os 20 e 38 anos, 50\% tinham o Ensino Médio Completo e $75 \%$ eram do sexo masculino. Aproximadamente $67 \%$ entrevistados estavam em regime de tratamento intensivo no serviço, $50 \%$ dos usuários tinham o tempo de uso entre um e dois anos, cerca de $67 \%$ com número de internações entre duas e três vezes e 58\% apresentaram problemas com a justiça.

Os usuários de crack avaliaram que a participação dos familiares no grupo era importante, pois se apresentava como fonte de escuta, possibilitando que os mesmos expressassem seus sentimentos frente aos desafios que a adição ao crack gerava no meio familiar.

Eu acho que é bom para a minha mãe esse grupo, porque ela tem com quem conversar. (U5)

Pela minha mãe, ela vem sempre. Está sempre aqui no CAPS nos grupos. (U12)

Os grupos de apoio aos familiares contribuíam para uma mudança significativa nos modos de viver no domicilio, na avaliação dos usuários. Essas alterações ocorriam a partir do momento em que o familiar mudava sua forma de tratar o usuário, tendo mais paciência, melhor manejo ao lidar com o usuário no seu cotidiano.

Aí a mãe começou a ir aos grupos e passou a ter mais paciência comigo. Ela passou a sentar e a conversar comigo. Hoje ela se preocupa mais. (U8)
É acho que os grupos ajudam bastante minha mãe. Mudou, porque às vezes quando eu vou lá, ela pega e vê e me bota pra dentro de casa. Mudou até o jeito de ser dela. (U11)

Eu acho bons os grupos, pelo menos ela aprende um pouco a tratar comigo. A mãe tá mais calma. Ela não bate mais boca comigo, porque antigamente ela batia muita boca, brigava. Agora não, ela me deixa ficar batendo boca sozinho. (U12)

Na avaliação dos entrevistados, a participação nos grupos de famílias propiciava maior envolvimento da família no tratamento do usuário de crack. Essa repercussão gerava no usuário conforto e satisfação, tendo em vista que o familiar demonstrava interesse pelo cuidado e reabilitação.

Os usuários avaliaram que havia um vínculo consolidado entre CAPS e família e um benefício gerado nessa inserção, quando citaram o tempo em que seus familiares estavam inseridos no grupo de apoio no serviço.

Há um ano minha mãe e irmã vem nos grupos do CAPS. Primeiro eu vim, depois elas. (U1)

Minha mãe participa a uns oito meses dos grupos aqui. (U10)

Os usuários de crack sentiam-se cuidados quando percebiam que sua família estava participando dos grupos e das atividades do serviço, no entanto ficavam incomodados quando estas não conseguiam fazerem-se presentes nestes espaços.

Às vezes, alguns grupos, minha mãe não estava vindo. Isso eu achava errado dela. Eu até falei isso para minha psicóloga aqui. Tem uns grupos que ela não veio, eu até fiquei chateado e falei: "A senhora não tá se importando em me ajudar?" Ela disse que não podia estar sempre vindo aqui, pois trabalha. (U3)

Os usuários de crack avaliaram que o número de participações semanais não era o bastante, pois acreditavam que, quanto maior a frequência das famílias nas reuniões, maiores seriam os benefícios gerados para o familiar e para eles próprios. 
Eu, particularmente, acho que seria melhor duas vezes por semana. Seria um dia a mais pra entenderem ainda mais minha situação. (U2)

No meu pensamento acho que umas duas ou três vezes por semana. Se um dia eu chegar drogado, a mãe sabe o que fazer ou alguma coisa parecida assim. (U4)

A mãe conversa bastante sobre drogas comigo, sobre o que aconteceu, mas seria melhor se tivesse um pouquinho mais de reuniões porque daí ela teria como falar as coisas certas pra mim. (U8)

Mesmo com a diversidade de horários (cinco horários divididos nos três turnos) oferecidos pelo CAPS AD muitos familiares participavam uma vez por semana, frequência que muitos usuários avaliaram como insuficiente. Faz-se importante salientar que os familiares possuem suas atividades profissionais e pessoais, o que certamente implicará na frequência deles nos grupos.

Este estudo avaliativo aponta que o cotidiano conturbado, repleto de discussões, brigas, xingamentos, e o cuidado intensivo dos familiares em relação aos usuários de crack geravam nas famílias sobrecargas físicas e emocionais. Essa sobrecarga fazia com que o familiar chegasse ao limite de uma agressão física no usuário.

É bom para a família saber como lidar com a gente, porque a minha mãe antes não sabia lidar comigo. Ela ficava em "estado de nervo", uma vez ela me "esguelou" e tudo, porque ela não sabia mais o que fazer comigo. (U7)

Os grupos instrumentalizam as famílias a compreenderem a dependência química como uma doença e que, por isso, os usuários devem ser tratados como doentes, conforme avaliaram os entrevistados.

Antes de a minha família estar dentro do CAPS, dentro do grupo do apoio, eles não entendiam que eu era um doente, que a minha doença era lenta, progressiva. Hoje, minha família lê, minha família pega informação. Só dentro do grupo de apoio, é que eles criam essa estrutura. (U1)
Esses grupos dão tranquilidade para meu pai. Ele sabe que eu estou sofrendo através dos grupos. Nós conversamos muito agora. (U2)

O meu filho de 16 anos acabou entendendo que era uma doença que eu tinha, porque antes eles viam como "senvergonhismo". Que eu era sem vergonha, que eu não tinha vergonha na cara. Aí ele percebeu que é uma doença por causa dos grupos. (U9)

\section{DISCUSSÃO}

O papel que as famílias desempenham nos cuidados ao seu usuário de crack vem sendo reconhecido como uma valiosa colaboração no seu tratamento e reabilitação, tendo em vista que os usuários evoluem melhor quando têm o apoio da família ${ }^{(4)}$. Nesse sentido, o grupo de apoio possibilita um momento no qual os familiares podem compartilhar os problemas entre si, sendo esta uma forma de se sentirem incluídos no tratamento, colaborando na superação das dificuldades ${ }^{(11-12)}$ inerentes ao uso de crack.

A participação dos familiares nos grupos ofertados pelo CAPS AD foi avaliada pelos usuários de crack como um aspecto importante para o seu tratamento, uma vez que instrumentaliza os familiares na mudança de atitude frente ao reconhecimento da doença e aos estágios de recuperação, implicando diretamente na forma que age perante o tratamento.

A necessidade de adaptação à nova situação, o estigma social, a dependência e as implicações de sua cronicidade produzem sobrecarga, conflitos, sentimentos de incredulidade, perda do controle e medo, além do desgaste biopsicológico da família ${ }^{(12-13)}$. A família, por sua vez, em um primeiro momento, espera que o usuário busque o tratamento no CAPS para só depois recorrer à ajuda para si própria nos grupos, em uma atitude de incredulidade e desconfiança do processo de reabilitação e dos benefícios dos grupos.

Dessa forma o núcleo familiar torna-se corresponsável no auxílio para o enfrentamento das situações de dependência química ${ }^{(6)}$. Assim, melhorar as relações familiares através dos grupos de apoio é resgatar a família novamente como um fator de proteção na prevenção de recaídas e na continuidade do tratamento, estimulando 
a motivação do usuário a seguir o plano de cuidados construído pelos profissionais do CAPS AD. Cabe, ainda, ressaltar que a família é considerada a rede de apoio mais próxima ${ }^{(7,14)}$ do usuário de crack. Assim, a motivação e a adesão do usuário de crack ao tratamento dependem muito da aceitação e apoio da família.

Quando um ser humano é diagnosticado com transtorno mental, sabemos que a evolução do tratamento depende muito da aceitação e apoio da família e da inclusão social do mesmo, pois somente com o sentimento de pertencimento e autonomia é que o ser humano poderá resgatar sua autoestima, autoimagem e estabelecer-se como sujeito de sua própria história(15:4).

Nesse sentido, as relações entre usuário e seus familiares tornam-se tensas e fragilizadas, pois devido ao caráter incontrolável do consumo, o usuário passa a cometer pequenos furtos na sua própria residência ou mesmo na rua ${ }^{(4,16)}$. Além disso, pode surgir a sobrecarga física e emocional, sentimentos de impotência e fracasso, o que leva as famílias à necessidade de suporte terapêutico, devido ao ambiente familiar ser permeado pela violência no domicílio.

Através da participação dos grupos, as famílias adquirem conhecimento sobre a adição ao crack e sobre o sofrimento do usuário, o que gera maior entendimento e harmonia no relacionamento, contribuindo para um clima de diálogo e de trocas mútuas no domicílio.

O grupo fortalece os vínculos familiares, oferecendo apoio emocional e informações/ orientações sobre a droga e dependência química do crack. Estes grupos possibilitam a percepção da situação real que estão vivendo, por meio do conhecimento de dados mais concretos, diminuindo as fantasias, ajudandoos no enfrentamento da crise vivenciada ${ }^{(12)}$. Assim, o grupo ajuda o familiar a compreender a dependência química como geradora de sofrimento também para o usuário e que por isso ele necessita de cuidados e apoio.

O grupo instrumentaliza a família com informações sobre os efeitos da droga no organismo do usuário, favorecendo o entendimento sobre o comportamento do usuário e, consequentemente, mudando sua concepção na forma de lidar com essas mudanças no domicílio(7). Paciência e diálogo são mudanças positivas geradas pela participação no grupo de familiares na avaliação dos usuários de crack, propiciando maior motivação para adesão e a prevenção de recaídas.

Os grupos de apoio com a finalidade de promover troca de experiência e suporte para cuidadores têm se mostrado uma prática bastante eficaz, com resultados benéficos tanto para os cuidadores quanto para o paciente cuidado, instrumentalizando os cuidadores na adoção de estratégias para prevenir possíveis estresses e desgastes $^{(7,13-14)}$.

A avaliação dos entrevistados não levou em conta a disponibilidade de tempo dos seus familiares, tendo em vista que muitos trabalhavam. Aumentar a frequência dos grupos de apoio às famílias no CAPS AD III implicaria na necessidade de um acordo prévio entre todos os participantes. Se, por um lado, a presença constante da família é muito importante para o usuário de crack, por outro é necessário ter cautela em não sobrecarregar essas famílias para que elas não sofram com maior desgaste físico e emocional.

As famílias dos dependentes químicos representam a principal rede de apoio do indivíduo, e, se bem acompanhadas terapeuticamente, tornam-se mais bem preparadas para enfrentar a situação $\mathrm{O}^{(14,17)}$. Assim, o aumento semanal da participação nos grupos de apoio dependerá da disponibilidade, da demanda e da indicação da equipe do serviço, que avaliará a existência ou não dessa necessidade.

\section{CONSIDERAÇÕES FINAIS}

Ao longo do processo de investigação e pela análise e discussão dos resultados, buscou-se aproximar de uma resposta para o problema de pesquisa e, desse modo, conhecer a avaliação dos usuários de crack sobre os grupos de familiares em um CAPS AD III do Sul do país.

No decorrer deste trabalho evidenciou-se, a partir da avaliação dos usuários entrevistados, que a participação dos familiares no grupo é de extrema importância, pois esse espaço apresentase como fonte de escuta, de esclarecimentos e de desabafo frente aos desafios que a adição ao crack gera no meio familiar, contribuindo positivamente para uma mudança nos modos de 
lidar com o usuário no domicílio.

Essas alterações ocorrem a partir do momento em que o familiar que frequenta o grupo muda sua maneira de tratar o usuário, tendo mais paciência e melhor manejo ao lidar com ele no seu cotidiano. No entanto, os participantes avaliaram que o número de encontros por semana dos grupos de familiares era insuficiente, pois acreditavam que, quanto mais encontros houver, maiores serão os benefícios gerados tanto ao familiar, quanto a eles próprios.

Faz-se necessário ressaltar as limitações deste estudo, considerando que o processo hermenêutico dialético é dinâmico, havendo dados que poderiam ter sido mais bem contemplados nas discussões do processo avaliativo. Porém, o fator tempo de realização do estudo interferiu no processo da pesquisa.

Este estudo avaliativo deu voz aos usuários de crack quanto aos grupos de familiares no CAPS AD, ficando evidente a importância desse dispositivo no tratamento dos usuários. Sugere-se que os serviços substitutivos sigam proporcionando horários diversificados de grupos de familiares para fidelizar as famílias nestes espaços, promovendo a continuidade do tratamento e os efeitos benéficos para o usuário de crack.

\section{REFERÊNCIAS}

1. Oliveira GP. Crack e recaída: os principais motivos que levam os usuários de crack recaírem após tratamento para dependência química [tese]. Porto Alegre (RS): Faculdade de Medicina, Universidade Federal do Rio Grande do Sul; 2011 [acesso em 3 abr 2013]. 22 p. Disponível: http://www.lume.ufrgs.br/bitstream/ handle/10183/34045/000789984.pdf

2. Salgado N. A. A pedra não para: um estudo sobre a cracolândia na cidade de Belo Horizonte/MG. Rev. UFMG [Internet] 2013;20(1) [acesso em 10 jun 2014]. Disponível: https://www.ufmg.br/revistaufmg/ downloads/20/13-a_pedra_nao_para_nayara\%20 salgado.pdf

3. Ministério da Saúde (BR). Coordenação Nacional de Saúde Mental, Álcool e outras Drogas. O crack: como lidar com este grave problema. [Internet]. Brasília; 2009 [acesso em 23 mar 2013]. Disponível: http:// portalses.saude.sc.gov.br/index.php?option =com docman\&task=doc_download\&gid=3151\&ltemid=85

4. Seleghim MR, Marangoni SR, Marcon SS, Oliveira
MLF. Family ties of crack cocaine users cared for in a psychiatric emergency department. Rev. Latino-Am. Enfermagem. [Internet] 2011;19(5) [cited 5 abr 2013]. Avaliable from: http://www. scielo.br/scielo.php?script=sci_arttext\&pid=S0104$11692011000500014 \& \operatorname{lng}=\mathrm{en} \& \mathrm{nrm}=\mathrm{iso} \& \operatorname{lng}=\mathrm{en} \& \mathrm{O}$ RIGINALLANG=en

5. Filzola CLA, Tagliaferro P, Andrade AS, Paravini SCl, Ferreira NMLA. Alcoolismo e família: a vivência de mulheres participantes do grupo de autoajuda Al Anon. J. bras. psiquiatr. [Internet] 2009;58(3) [acesso em 15 mar 2013]. Disponível: http://dx.doi. org/10.1590/S0047-20852009000300007

6. Oliveira EB, Medonça JLS. Family member with chemical dependency and consequent overburden suffered by family: descriptive research. Online Braz J Nurs. [Internet] 2012;11(1) [cited 1 jun 2014]. Avaliable from: http://www.objnursing.uff.br/index.php/nursing/ article/view/3480/html

7. Alvarez SQ, Gomes GC, Oliveira AMN, Xavier DM. Grupo de apoio/suporte como estratégia de cuidado: importância para familiares de usuários de drogas. Rev. gauch. enferm. [Internet] 2012;33(2) [acesso em 21 abr 2014]. Disponível: http://www. seer.ufrgs.br/RevistaGauchadeEnfermagem/article/ viewFile/24646/19499

8. Duarte MLC. Familiares dos usuários de crack em um CAPS AD III: avaliação das necessidades de cuidados [tese]. Porto Alegre (RS): Escola de Enfermagem, Universidade Federal do Rio Grande do Sul; 2013. 204 p.

9. Guba E, Lincoln Y. Fourth generation evaluation. Newbury Park (CA): Sage; 1989.

10. Ministério da Saúde (BR). Conselho Nacional de Saúde. Resolução 196 de 10 de outubro de 1996: diretrizes e normas regulamentadoras de pesquisas envolvendo seres humanos. Brasília; 1996.

11. Benevides DS, Pinto AGA, Cavalcante CM, Jorge MSB. Cuidado em saúde mental por meio de grupos terapêuticos de um hospital-dia: perspectivas dos trabalhadores de saúde. Interface, Comun., Saúde, Educ. [Internet]. 2010;14(32) [acesso em 20 mar 2013]. Disponível: http://dx.doi.org/10.1590/S141432832010000100011

12. Cassol PB, Terra MG, Mostardeiro SCTS, Gonçalves MO, Pinheiro UMS. Tratamento em um grupo operativo em saúde: percepção dos usuários de álcool e outras drogas. Rev. gauch. enferm. [Internet] 2012;33(1) [acesso em 13 ago 2013]. Disponível: http://www.scielo.br/scielo.php?pid=S1983 14472012000100018\&script=sci_arttext

13. Gabatz RIB, Schmidt AL, Terra MG, Padoin SMM, Silva AA, Lacchini AJB. Percepção dos usuários de crack em relação ao uso e tratamento. Rev. gauch. enferm. [Internet] 2013;34(1) [acesso em 11 mai 
2014]. Disponível: http://dx.doi.org/10.1590/S198314472013000100018

14. Maciel LD, Zerbetto SR, Filizola CLA, Dupas G, Ferreira NMLA. Consequências e dificuldades da dependência química no âmbito familiar: uma revisão de literatura. Rev. APS [Internet] 2013;16(2) [acesso em 11 dez 2013]. Disponível: http://aps.ufjf.emnuvens. com.br/aps/article/view/1809/721

15. Santos TC, Carrapato JL. A importância da família na qualidade de vida das pessoas com doença mental. $\checkmark$ Encontro de iniciação científica, IV Encontro de extensão universitária e I Encontro de iniciação científica p/ o ensino médio. [Internet] 2009; 5(5) [acesso em 15 nov 2014]. Disponível: http:// intertemas.unitoledo.br/revista/index.php/ETIC/article/ viewFile/2195/2343

16. Magalhães DEF, Silva MRS. Cuidados requeridos por usuários de crack internados em uma instituição hospitalar. Reme, Rev. Min. Enferm. [Internet] 2010;14(3) [acesso em 15 dez 2013]. Disponível: http:// www.enf.ufmg.br/site_novo/modules/mastop_publish/ files/files_4d3079563e899.pdf

17. Soccol KLS, Terra MG, Ribeiro DB, Teixeira JKS, Siqueira DF, Mostardeiro SCTS. O cotidiano das relações familiares com indivíduo dependente químico. Cogitare enferm. [Internet] 2014;19(1) [acesso em 17 out 2014]. Disponível: http://ojs.c3sl.ufpr.br/ojs/ index.php/cogitare/article/view/35967/22176 